\title{
ATITUDES DOS ENFERMEIROS COM RELAÇÃO AO ALCOOLISMO: UMA AVALIAÇÃO DE CONHECIMENTOS.
}

\author{
NURSES' ATTITUDES TOWARD ALCOHOLISM: A KNOWLEDGE EVALUATION \\ ACTITUDES DE LOS ENFERMEROS RESPECTO AL ALCOHOLISMO: UNA EVALUACIÓN DE \\ CONOCIMIENTOS
}

\section{Sandra C. Pillon ${ }^{1}$}

RESUMO: Este artigo tem como objetivo avaliar os conhecimentos adquiridos sobre os problemas relacionados ao uso de álcool e aos usuários pelos enfermeiros (alunos, docentes e enfermeiros assistenciais) de uma universidade publica brasileira durante a formação educacional. Para esta pesquisa foi elaborado um questionário que contém avaliações sobre os conteúdos elementares e específicos relacionados aos problemas do alcoolismo, baseado no NEADA FACULTY SURVEY (PROJECT NEADA Nursing Education in Alcohol and Drug Education). Concluímos que existe uma deficiência na educação formal, onde encontramos em grande proporção que os enfermeiros obtiveram pouco ou nenhum conhecimento em nível semi-especializado ou especializado sobre o tema álcool e suas conseqüências, 0 que nos leva a discutir a necessidade de uma maior oferta de treinamentos nessa área.

PALAVRAS-CHAVE: Educação em Enfermagem; Transtornos Relacionados ao Uso de Álcool; Conhecimentos, Atitudes e Práticas em Saúde.

ABSTRACT: This article aims to evaluate what knowledge nurses (students, teachers and clinical nurses) from a Brazilian public university acquired during their education with respect to problems related to alcohol use and users. A questionnaire was elaborated with evaluations of elementary and specific contents related to alcoholism problems, based on the NEADA FACULTY SURVEY (PROJECT NEADA Nursing Education in Alcohol and Drug Education). We concluded that there is a gap in formal education, which frequently revealed that nurses obtained little or no semi-specialized or specialized knowledge about the subject of alcohol and its consequences. As a result, we discuss the need for greater training in this area.

KEY-WORDS: Nursing Education; Health Knowledge, Attitudes, Practice; Alcohol-Related Disorders.

RESUMEN: La finalidad de este artículo es evaluar los conocimientos que los enfermeros (alumnos, profesores y enfermeros asistenciales) de una universidad pública brasileña adquirieron durante la formación educacional, sobre problemas relacionados al uso de alcohol y a sus usuarios. Para esta investigación fue elaborado un cuestionario que contiene evaluaciones sobre los contenidos elementares y específicos relacionados a los problemas del alcoholismo, basado en el NEADA FACULTY SURVEY (PROJECT NEADA - Nursing Education in Alcohol and Drug Education). Concluímos que existe una deficiencia en la educación formal, donde encontramos frecuentemente que los enfermeros obtuvieron poco o ningún conocimiento semi-especializado o especializado sobre el tema alcohol y sus consecuencias, lo que nos lleva a discutir la necesidad de una mayor oferta de entrenamientos en esa área.

PALABRAS-CLAVE: Educación en Enfermería; Conocimientos, Actitudes y Práctica en Salud; Transtornos Relacionados con Alcohol.

1 Professor Doutor do Departamento de Enfermagem Psiquiátrica e Ciências da Escola de Enfermagem de Ribeirão Preto. Universidade de São Paulo - EERP.USP. Ribeirão Preto, SP email: pillon@eerp.usp.br 


\section{INTRODUÇÃo}

\section{Atitudes dos enfermeiros - revisão da literatura.}

Poucas pesquisas no Brasil tem sido direcionadas para a investigação das atitudes dos profissionais de saúde e em específico a dos enfermeiros em relação ao conhecimento adquirido em relação ao uso álcool e suas conseqüências. Embora os enfermeiros tenham uma formação educacional específica, suas atitudes podem não ser diferenciadas daquelas da população em geral.

Devido a sua atuação nos mais variados setores de saúde, os enfermeiros constituem um grande grupo de profissionais que freqüentemente depara-se com essa população. Situando-se, no entanto, numa posição privilegiada para identificar os pacientes usuários com problemas relacionados ao uso do álcool e desempenhar um papel fundamental nesta área. Uma visão negativa ou estereotipada talvez decorrentes das deficiências no currículo, são fatores que tem contribuído e dificultado na identificação e intervenção de enfermagem. Uma educação formal adequada deveria incluir conteúdos abordando a problemática do uso de álcool e drogas e suas conseqüências, visando identificação e intervenção mínima e ou encaminhamento adequado. A falha na formação educacional do enfermeiro em relação ao álcool, refletem talvez nas pesquisas, mostrando que quando se trata dos pacientes usuários de álcool, as atitudes e crenças dos enfermeiros em relação aos pacientes usuários são significativamente mais negativas e impregnadas de conteúdos morais do que aquelas que tem em relação a outros pacientes. Segundo GRIFFITHS \& PEARSON (1988), a omissão na identificação e intervenção precocemente, podem exacerbar os problemas do paciente usuário de álcool, tornando o seu prognóstico mais grave, chegando à sua cronificação. As atitudes e crenças negativas sobre informações de doenças relacionadas ao uso de álcool podem ser transformadas em reações negativas e de julgamento de valor a respeito do usuário, manifestas durante a intervenção de enfermagem.

Embora as organizações de saúde tenham oficialmente reconhecido o alcoolismo como doença, a partir da década de 50 , só recentemente ele tem sido mais aceito pela população em geral. Muitos enfermeiros e outros profissionais têm se mostrado relutante durante $\mathrm{a}$ assistência às necessidades desses pacientes, especificamente no reconhecimento do uso do álcool e dos problemas associados, bem como nas intervenções e orientações de encaminhamento (STARKEY, 1980).

Enfermeiros e profissionais de saúde têm apresentado atitudes negativas com relação ao uso de álcool e drogas e uma percepção pessimista quanto aos resultados do tratamento, sendo que as enfermeiras tendem a ser mais moralistas com relação aos bebedores e os percebem mais como fracos de caráter do que como doentes STARKEY (1980), além de pouco os tolerarem (SCHMID \& SCHIMID, 1973;
ROTHERAM, 1980). As atitudes negativas dos enfermeiros em relação ao alcoolismo interferem não apenas na detecção dos sintomas do alcoolismo, como também no encaminhamento dos identificados para o tratamento (ROSEMBAUM, 1977). Por outro lado, investiga-se pouco na história dos pacientes a ocorrência dos problemas relacionados ao beber e os bebedores quando identificados são avaliados como briguentos e manipuladores (FERNEAU \& MORTON, 1968; CORNISH \& MILLER 1976; KELLY \& MAY, 1982; MANSON \& RITSON 1984).

Os estudos realizados reforçam a idéia de que os conceitos dos profissionais e estudantes da área de saúde, em relação à etiologia do alcoolismo, não sofreram grandes mudanças com o decorrer do tempo. SULIVAN \& HALE (1978), observaram nesses profissionais o predomínio da crença numa etiologia psicológica e/ou biológica, não descartando totalmente o aspecto moral do problema.

PILLON et al, (1998), em um estudo no Brasil sobre atitudes dos enfermeiros com relação ao alcoolismo, investigando as características psicométricas entre três escalas sobre atitudes encontrou a influencia dos fatores morais concomitantes à uma mescla da etiologia psicológica e ou biológica da doença.

Lembrando TRAVELBEE (1971), a percepção do enfermeiro em relação ao paciente, "é o fator determinante da qualidade e quantidade dos cuidados de enfermagem que ela realizará', deste modo, o enfermeiro deveria avaliar suas próprias atitudes com relação ao alcoolismo para desenvolver cuidados mais humanos e sem julgamentos de valor ao paciente com problemas relacionados ao uso do álcool, pois as atitudes negativas podem afetar as habilidades na assistência prestada aos mesmos. O reconhecimento dos enfermeiros sobre essas atitudes poderá conduzilos a efetuar mudanças nos cuidados profissionais.

Diante da problemática identificada na literatura e da carência de estudos nacionais referentes ao tema, este estudo tem como objetivo avaliar os conteúdos teóricos referentes ao tema álcool recebido pelos enfermeiros (estudantes de enfermagem e enfermeiros assistenciais e docentes) durante sua formação educacional.

\section{METODOLOGIA}

Trata-se de um estudo descritivo, de investigação, que faz parte da dissertação de mestrado da autora principal na temática sobre as atitudes dos enfermeiros relacionados ao alcoolismo.

\section{Amostra e Local}

A amostra foi constituída por 512 (85\%) participantes dos quais: estudantes de enfermagem, enfermeiros (enfermeiros assistências e docentes de enfermagem) do Hospital São Paulo e Departamento de Enfermagem da Universidade Federal de São Paulo - UNIFESP/EPM. 
O instrumento de coleta de dados constitui-se de um questionário individual, estruturado com perguntas e respostas fechadas. Esse instrumento foi elaborado a partir da experiência profissional em trabalhar nesta área, associado à tradução do NEADA FACULTY SURVEY (PROJECT NEADA - Nursing Education in Alcohol and Drug Education). O roteiro da entrevista conduzia a questões, dividido em : 1) dados pessoais do sujeito; 2) Roteiro específico, uma investigação dos conteúdos sobre o uso do álcool e problemas relacionados, ministrados durante formação educacional; com respostas fechadas solicitando que assinale se obteve ou não o conteúdo e em qual momento de sua formação acadêmica.

A coleta de dados, seguiu algumas etapas: construção do questionário, avaliação da tradução por 2 juizes (um brasileiro e um britânico), autorização do Comitê de Ética de Pesquisas Clínicas "in Anima Nobili" da Escola Paulista de Medicina - UNIFESP e Diretoria de Enfermagem do Hospital, esclarecimento sobre a pesquisa e garantia do anonimato ao sujeito, um estudo piloto foi realizado, onde possibilitou discutir possíveis dificuldades sobre o questionário. Os enfermeiros foram contatados no próprio local de trabalho, onde foram convidados a participar do estudo pelo autor principal, mediante o questionário a ser preenchido posteriormente. Os questionários preenchimento foram recolhidos após um período de 24 horas.

\section{Critério de classificação das variáveis}

Foi utilizado um questionário contendo 18 avaliações que identificam os conteúdos abordando problemas relacionados ao uso do álcool, populações de risco e a assistência de enfermagem a essa população. As variáveis foram classificadas em 3 grupos de 6 variáveis, de acordo com os critérios estabelecidos pelos autores, representado na Figura 1 e respectivos, informações referentes aos conteúdos abordando os tipos de problemas (por ex.: problemas de saúde física, mental e social) relacionados ao uso do álcool, que o entrevistado recebeu durante sua formação lato senso. Para todos os grupo foram considerado "nenhuma informação" para as variáveis respondidas, não recebi este tipo de informação e ou quando havia apenas 1 resposta assinalada positiva (em apenas um momento de sua formação lato senso); "Poucas informações" respondidas positivamente até 3 variáveis apenas na graduação e por fim "muitas informações" quando respondida 4 ou mas variáveis positivas

\section{RESULTADOS}

O questionário foi distribuído a 603 sujeitos, dos quais $512(85 \%)$ da população total responderam-o por completo, os demais (15\%) foram perdidos ou retornados completamente em branco, sendo justificados pelos enfermeiros com falta de tempo para respondê-lo, ou por não lembrar onde deixou - o, mesmo assim foi oferecida uma segunda cópia do mesmo. Da amostra, $52 \%$ eram enfermeiros formados, $48 \%$ estudantes da graduação em enfermagem. Entre os estudantes de graduação de enfermagem $\mathrm{N}=248$ (primeiro ano 25\%, segundo ano $30 \%$, terceiro ano $22 \%$, quarto ano $23 \%$ ) e entre os enfermeiros $\mathrm{N}=264$ (docentes de enfermagem $28 \%$, e enfermeiros assistenciais 72\%). Da amostra eram 93 $\%$ mulheres, com idade média de 21 anos entre os estudantes ( $D p=2,58$ anos, Min 17 e Máx 37 anos) e enfermeiros idade média 22 anos ( $D p=8$ anos, Min 22 e Máx. 60 anos). Tempo de formado entre os enfermeiros em média de 9 anos (Dp = 9 anos, Min 1 - Máx. 36 anos). Sendo que setenta e dois porcento dos enfermeiros eram formados em universidades públicas.

Tabela 1. Perfil dos estudantes de graduação de enfermagem e enfermeiros formados (enfermeiros e docentes) em número e porcentagem.

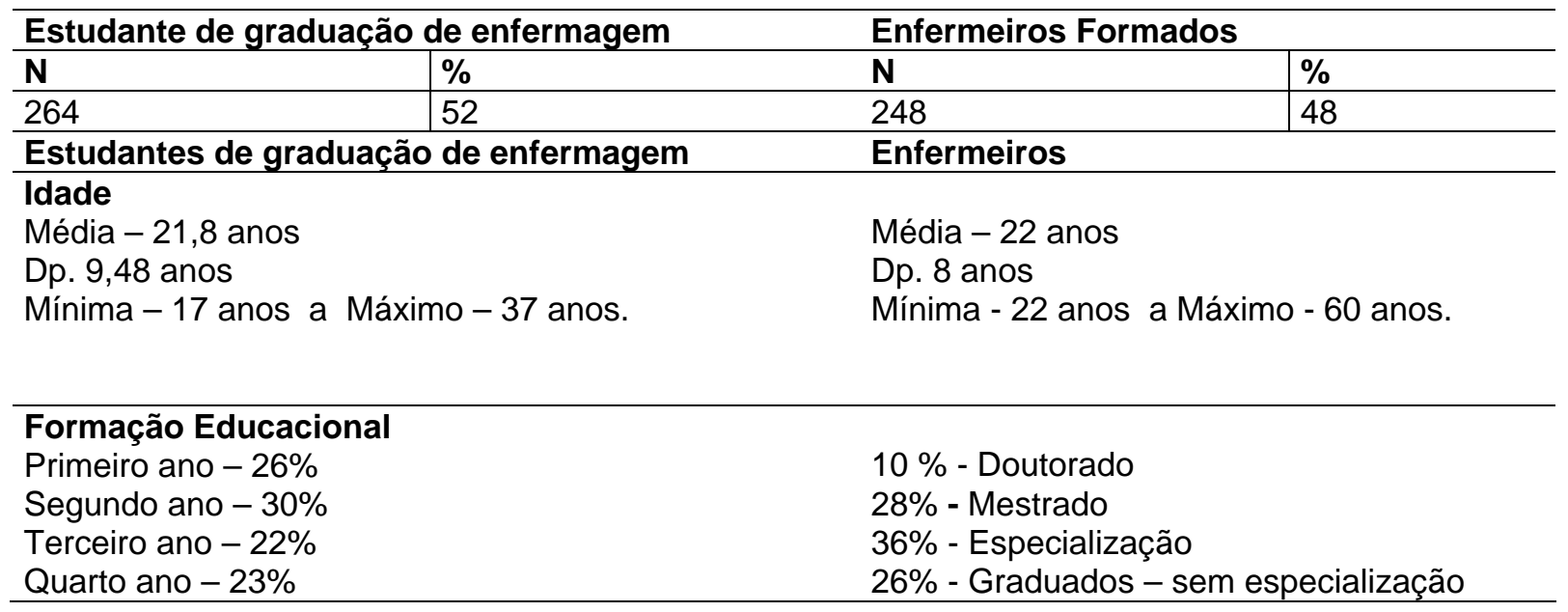


Figura 1: Distribuição das respostas apresentadas em porcentagem dos conteúdo abordados sobre problemas (fìsicos, famiiares e sociais) relacionados ao uso do álcool, $(\mathrm{N}=512)$

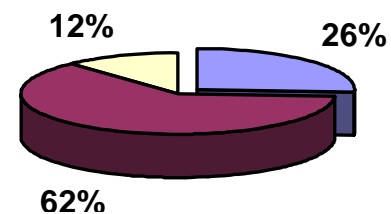

A Figura 1 mostra que $62 \%$ dos sujeitos receberam conteúdos abordando os problemas relacionados ao uso do álcool durante a formação lato senso, isto significa uma proporção de 1:4 não receberam nenhuma formação a esse respeito, e a proporção de 2:3 receberam poucas informações sobre os principais problemas relacionados ao uso do álcool o que corrobora com a literatura internacional. Não diferentemente nos grupos seqüentes, quanto mais a especificidade do problema maior a quantidade de falta de informações. Com diferença significativa entre os grupos (enfermeiros formados e estudantes) $\mathrm{X} 2=118,12 \mathrm{p}<.000$.

Figura 2: Apresentação em porcentagem das respostas dos sujeitos sobre as abordagens referentes ao tipo de população usuária de álcool específica ou de risco. $(\mathrm{N}=512)$

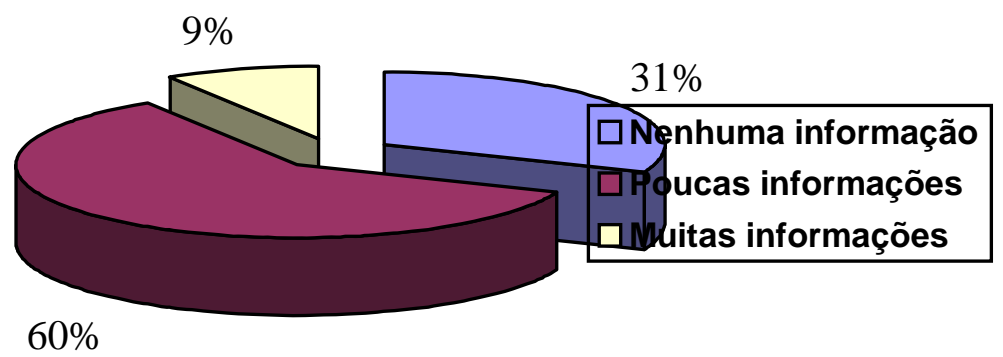

A Figura 2 apresenta os conteúdos sobre as populações específicas ou de riscos para o alcoolismo, como adolescentes, idosos, gestantes e outros. Isto representa uma proporção de 1:3 sujeitos receberam poucas informações, 3:5 sujeitos receberam poucas informações e 1:10 sujeitos receberam muitas informações sobre esse tema. Com diferença significativa entre os grupos (enfermeiros formados e estudantes) X2 = 69,94 p.<.000.

Figura 3: Distribuição das respostas em porcentagem das abordagens referentes a Assistência de Enfermagem prestada aos pacientes usuários de álcool, $(\mathrm{N}=512)$

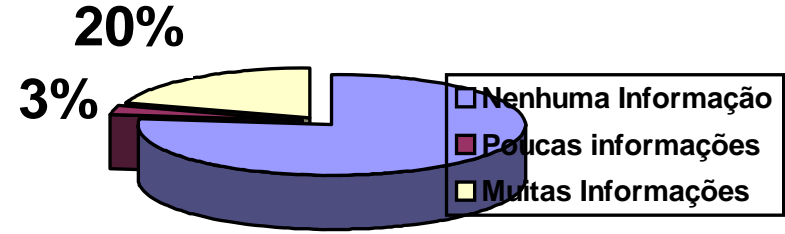

$77 \%$
A Figura 3 apresenta a abordagem recebida referente a assistência de enfermagem a essa população. Representado na proporção de 4:5 sujeitos que não receberam nenhuma informação, 1:5 receberam poucas informações, e 1:10 receberam muitas informações. Com diferença significativa entre os grupos (enfermeiros formados e estudantes) $\times 2=$ $17,40 \mathrm{p}<.000$.

CONCLUSÃO 
Em muitos serviços de saúde onde o enfermeiro oferece cuidados de enfermagem, ele exerce uma função fundamental em identificar e talvez iniciar uma intervenção terapêutica aos pacientes com problemas relacionados ao uso de álcool ou pelo menos encaminhar a um serviço apropriado. Infelizmente a literatura tem mostrado que além das atitudes negativa dos enfermeiros frente ao alcoolismo e uma deficiência na educação formal.

Este estudo corrobora com a literatura, a qual mostra uma deficiência na educação formal, onde observamos em grande proporção que os enfermeiros obtiveram pouco ou nenhum conhecimento sobre o tema álcool e suas conseqüências.

Existe a necessidade de incluir treinamentos formais e informais de acordo com os níveis básicos a especializados, que vão desde a identificação do uso abusivo em toda as populações, até o aprendizado de técnicas de intervenções breves mais específicas, abordagens iniciais e aconselhamentos sem julgamentos e mesmo um simples encaminhamentos à especialidade.

Este estudo tem implicações importantes para reflexões sobre os conteúdos disponíveis na grade curricular dos cursos de graduação de enfermagem, visando a formação educacional do enfermeiro e também de treinamentos básicos e continuados nessa área. $\mathrm{O}$ enfermeiro se bem treinado pode oferecer métodos alternativos que vão de encontro às necessidades de saúde.

As principais questões profissionais são: 1aproveitar as oportunidades que existem em oferecerem cuidados a esta população, 2- responder as necessidades, pela educação formal sobre este importante problema de saúde. Embora algumas escolas de enfermagem tenham iniciado abordagens sobre os conteúdos de álcool e drogas nos currículos, ele pode ir de encontro às mudanças que nada mais intervir efetivamente nas necessidades dos cuidados de saúde do século 21 .

\section{REFERÊNCIAS BIBLIOGRÁFICAS}

CORNISH, R.D., MILLER, M.V. Attitudes of registered nurses toward the alcoholic. Journal of Psychiatric Nursing, n.14, p.19-22, 1976.

FERNEAU, E.W., MORTON, E.L. Nursing personnel and alcoholism. Nursing Research, n 17, p.174-7, 1968 GRIFFITHS, R., PEARSON, B. Working with Drug Users. Wildwood House, Hants, 1988.

KELLY, M.P, MAY D. Good and bad patients: a review of the literature and theoretical critique. Journal of Advanced Nursing, n.7, 147-6, 1982.

MANSON, L., RITSON, B. Alcohol and Health. A hand book for nurses, midwives and health visitors. Medical Council on Alcoholism, London 1984.

PILLON, S.C., LARANJEIRA, R.R., DUNN, J. Nurses Attitudes Towards Alcoholism: Factor analysis of three commonly used scales. Revista São Paulo Medical Journal, v.116, n.2, p.1661-6, 1998.

ROSEMBAUM, P.D. Public health nurses in the treatment of alcoholic abusers. Canadian Journal of Public Health, n.68, 503-8, 1977.

ROTHERAM, F. Nurses and alcohol problems. Nursing Times 1980, 76, 2197-8.

SCHMID, J., SCHIMID, D. Nursing students' attitudes towards alcoholics Nurs Res 1973, 22: 246-248.

SULLIVAN, E.J; HALE, R.E. Nurses' beliefs about the etiology and treatment of alcohol abuse: a national study. J Stud Alcohol., v. 48, n. 5, p. 456-460. 1978.

STARKEY, P.J. Nurses' attitudes toward alcoholism. AORN Journal, 31(5), 819-832. 1980.

TRAVELBEE, J. Interpersonal aspects of nursing. Philadelphia; Davis 1971.

Texto recebido em 17/11/2005.

Publicação aprovada em 10/12/2005 